\title{
ERRATUM
}

\section{A points-based algorithm for prognosticating clinical outcome of Chiari malformation Type I with syringomyelia: results from a predictive model analysis of 82 surgically managed adult patients}

TO THE READERSHIP: A few errors appeared in the article by Thakar et al. (Thakar S, Sivaraju L, Jacob $\mathrm{KS}$, et al. A points-based algorithm for prognosticating clinical outcome of Chiari malformation Type I with syringomyelia: results from a predictive model analysis of 82 surgically managed adult patients. J Neurosurg Spine. 2018;28[1]:23-32).

In Table 2, a few of the listed percentage values were erroneous, and the value for "Obex position relative to FM, mm" was incorrect; the correct value is " $7.96 \pm 3.12$." The corrected table appears here. 2020 .

The article has been corrected online as of May 22,

Sumit Thakar, $\mathrm{MCh}$

Sri Sathya Sai Institute of Higher Medical Sciences, Bangalore, India

\section{INCLUDE WHEN CITING}

Published online May 22, 2020; DOI: 10.3171/2020.5.SPINE17264a.

CAANS 2020, except where prohibited by US copyright law
TABLE 2. Radiological variables of the study cohort

\begin{tabular}{|c|c|}
\hline Variable & Value \\
\hline \multicolumn{2}{|l|}{ Syrinx location, n (\%) } \\
\hline Cervical & $8(14.03)$ \\
\hline Cervicothoracic & $38(66.66)$ \\
\hline Thoracic & $1(1.75)$ \\
\hline Holocord & $10(17.54)$ \\
\hline \multicolumn{2}{|l|}{ Syrinx type, n (\%) } \\
\hline Central & $6(10.53)$ \\
\hline Enlarged & $45(78.94)$ \\
\hline Deviated & $6(10.53)$ \\
\hline Scoliosis, $n(\%)$ & $17(29.82)$ \\
\hline Syrinx levels & $12.23 \pm 4.67$ \\
\hline Tonsillar herniation & $18.53 \pm 6.78$ \\
\hline Syrinx/cord ratio & $0.75 \pm 0.17$ \\
\hline 4th ventricle width, $\mathrm{mm}$ & $12.48 \pm 4.99$ \\
\hline C-2 retroversion, deg & $104.69 \pm 8.52$ \\
\hline C-2 retroflexion, deg & $101.28 \pm 7.29$ \\
\hline Clivus-canal angle, deg & $135.29 \pm 10.98$ \\
\hline Cerebellomedullary angle, deg & $148.48 \pm 10.92$ \\
\hline $\mathrm{pB}-\mathrm{C} 2$ distance, $\mathrm{mm}$ & $4.54 \pm 2.31$ \\
\hline Obex position relative to $\mathrm{FM}, \mathrm{mm}$ & $7.96 \pm 3.12$ \\
\hline \multicolumn{2}{|l|}{ FM dimensions, mm } \\
\hline Anteroposterior, i.e., McRae line & $31.93 \pm 4.89$ \\
\hline Lateral & $28.01 \pm 3.82$ \\
\hline PFV/ICV ratio & $153.00 \pm 28.76$ \\
\hline L-line-bulbopontine sulcus distance, $\mathrm{mm}$ & $12.19 \pm 3.28$ \\
\hline L-line-FVV distance, $\mathrm{mm}$ & $21.50 \pm 5.49$ \\
\hline L-line-tonsillar tip distance, $\mathrm{mm}$ & $17.37 \pm 7.81$ \\
\hline M-line-bulbopontine sulcus distance, $\mathrm{mm}$ & $2.13 \pm 3.31$ \\
\hline M-line-FVV distance, $\mathrm{mm}$ & $22.41 \pm 6.01$ \\
\hline M-line-tonsillar tip distance, $\mathrm{mm}$ & $21.16 \pm 4.51$ \\
\hline C2-7 sagittal angle, deg & $13.48 \pm 12.48$ \\
\hline Cervical taper ratio & $-0.51 \pm 0.47$ \\
\hline
\end{tabular}

Values are shown as the mean \pm SD unless indicated otherwise. 